\title{
Binarization of Inhomogeneously Illuminated Images
}

\author{
Volodymyr V. Kindratenko, Boris A. Treiger, Piet J.M. Van Espen \\ Department of Chemistry, University of Antwerp (UIA), \\ Universiteitsplein 1, B-2610 Antwerpen-Wilrijk, Belgium
}

\begin{abstract}
Most grey level thresholding methods produce good results only when the illumination of the image is homogeneous. An automatic binarization technique suitable for images containing regions of different brightness is presented. It does not use image's grey level histogram as a source of information. Rather, the method is based on the transformation of the raster image in such a way that the transformed image can be easy thresholded. A comparison with histogram based threshold selection technique is given.
\end{abstract}

\section{Introduction}

Image analysis often involves the discrimination between "objects" and the background. Grey level thresholding (also known as binarization) technique has been proven a suitable tool for this purpose. Various approaches for the automatic selection of a threshold have been proposed. A survey of them can be found in [1].

In general, thresholding can be defined as mapping the grey scale into the binary set $\{0,1\}$ :

$$
S_{x y}=\left\{\begin{array}{lll}
0, & \text { if } & g_{x y}<T_{x y} \\
1, & \text { if } & g_{x y} \geq T_{x y}
\end{array}\right.
$$

where $S_{x y}$ is the value of the segmented image, $g_{x y}$ is the grey level of the pixel and $T_{x y}$ is the threshold value at the coordinates $(x, y)$. In the simplest cases $T_{x y}$ is coordinate independent and a constant for the whole image. It can be selected, for instance, on the base of the analysis of the grey level histogram (GLH). When the GLH has two pronounced maximum, which reflect grey levels of object(s) and background, it is possible to select a single threshold for the whole image. However, sometimes GLH has only one maximum. This can be caused, e.g., by inhomogeneous illumination of various regions of the image. In such cases it is impossible to select a single thresholding value for the whole image and local binarization technique [2] must be applied. General methods to solve the problem of binarization of inhomogeneously illuminated images, however, are not available.

The proposed technique, which works well in case of inhomogeneously illuminated images (similar to given in Figs 1a and 4a), is based on the application of discrete convolution filtering technique that produces a transformed image, which can be easy thresholded. The threshold value $T_{x y}$ in equation (1) for the transformed image is coordinate independent and equals to 1 . 


\section{Discrete Convolution Filtering Technique}

The descrete convolution of image $f$ with mask $h$ produces an image $g$ :

$$
g(x, y)=\sum_{i=-v}^{v} \sum_{j=-u}^{u} h(i, j) * f(x+i, \quad y+j)
$$

where $h$ is a $2 v+1$ by $2 u+1$ matrix. The output $g(x, y)$ at a point $(x, y)$ is given by a weighted sum of input pixels around $(x, y)$ where the weights are given by the $h(i, j)$. Equation (2) can be implemented by a series of shift-multiply-sum operations. The values of $h$ are referred to as the filter kernel [3]. The effect of convolution on the original image depends on the type of filter kernel used. It can have a smoothing effect (e.g., weighted mean filter), an edge enhancing effect (e.g., the Laplacian added back to the original image), etc. [4]. We use the descrete convolution technique to produce a "binarization" effect.

\section{Description of Algorithm}

The main idea of the proposed technique, as it was mentioned above, is to transform the grey level image in such a way that it will be possible to select a single threshold value $T_{x y}$ for the whole transformed image. The operation of transformation of the grey level image can be represented as the descrete convolution of grey level image with the filter kernel of a special form. This filter kernel is constructed according to the following rule: the elements of the kernel are equal to -1 if they belong to the first or last rows or the first or last columns, -2 if they are not defined yet and belong to the second or last but one rows or the second or last but one columns, and so on. The central element of the kernel equals minus the sum of all elements of the kernel plus an additional parameter $p$. We used filter kernels of size 5 by 5 and 7 by 7 . The filter kernel of the size 3 by 3 can be used, but results are slightly worse. Increasing of the size of the filter kernel does not lead to much better results and only increases computational time. Two examples of such kernels are given in Figs 2a and 5a.

The problem remains, however, how to define the parameter $p$. The higher it is, the more details will be lost. With small $p$ a lot of details in the image may not be enhanced. With $p=0$ the proposed filter produces edge enhancement. For decision making about a suitable numerical value of parameter $p$, the coefficient of correlation between original and convoluted images was used. It is calculated as follows:

$$
r=\frac{\operatorname{Cov}(X, Y)}{\sqrt{\operatorname{Var}(X)^{*} \operatorname{Var}(Y)}}
$$

where $X$ and $Y$ are the original grey level image and the image convolved using a value $p$ for the parameter, respectively. As shown in Figs $2 b$ and $5 b$, the correlation coefficient reaches a maximum at a certain $p$. The parameter $p$ corresponding to the maximal correlation can be used as a suitable one for the current image. 


\section{Results}

Figs 1a and 4a represent two images of the surface of material with a cell structure. Both images have inhomogeneously illuminated regions. The upper left corners of both images are much darker than the centres. The grey level histograms of both images (Figs $1 \mathrm{~b}$ and $4 \mathrm{~b}$, respectively) have no pronounced maxima related to "objects" and/or background. A single threshold value selection for the whole image does not allow to obtain a binary image (see Figs $3 \mathrm{a}$ and 6a, respectively). The proposed technique gives binary images shown in Figs $3 \mathrm{~b}$ and $6 \mathrm{~b}$. Dark spots can be observed on the binarized images. They appear as a result of noise and can be removed by noise filtering techniques [4].

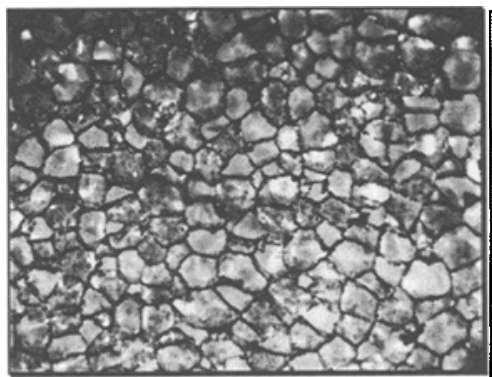

$\mathbf{a}$

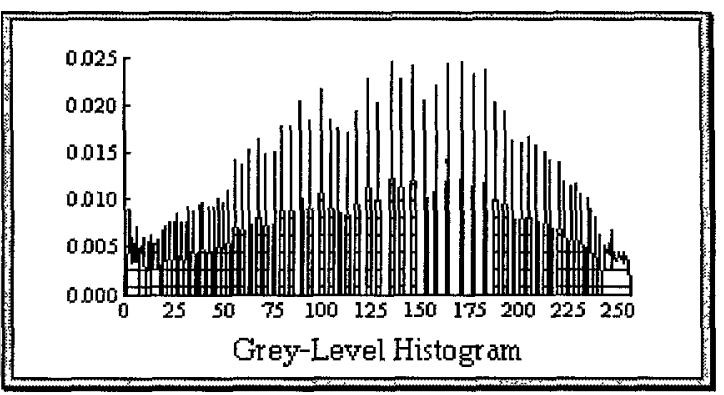

b

Fig. 1. Original image (a) and its grey level histogram (b).

$$
\left(\begin{array}{ccccc}
-1 & -1 & -1 & -1 & -1 \\
-1 & -2 & -2 & -2 & -1 \\
-1 & -2 & 32+p & -2 & -1 \\
-1 & -2 & -2 & -2 & -1 \\
-1 & -1 & -1 & -1 & -1
\end{array}\right)
$$

a

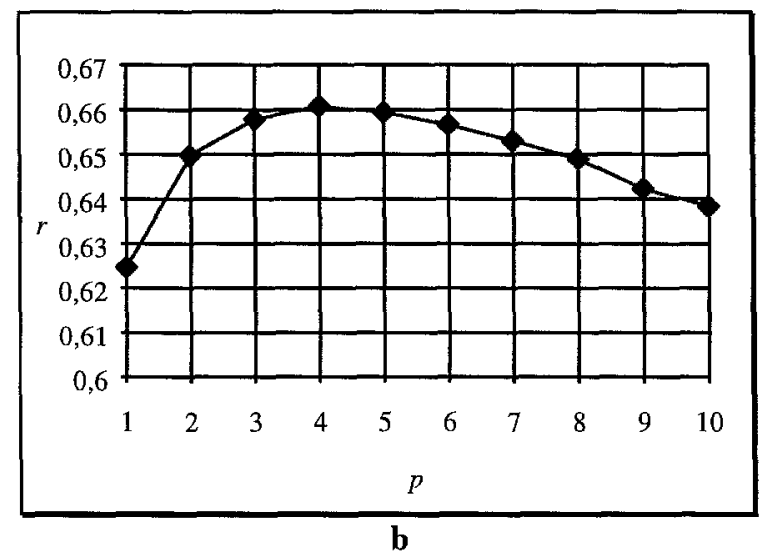

Fig. 2. Convolution kernel (a) and graph with the coefficient of correlation between original (Fig. la) and convoluted (with kernel from Fig. 2a for $p=1,2, \ldots, 10$ ) images vs. parameter $p$ (b). 


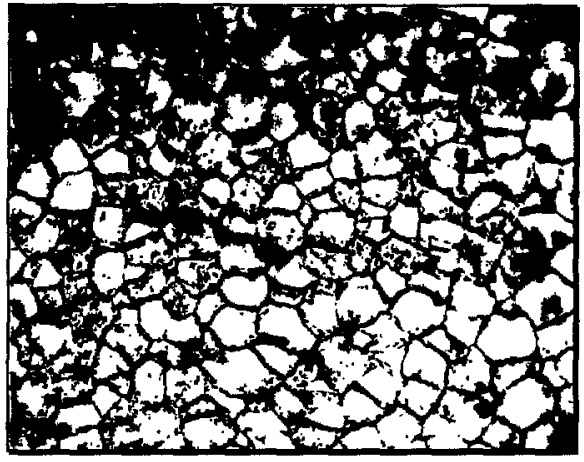

a

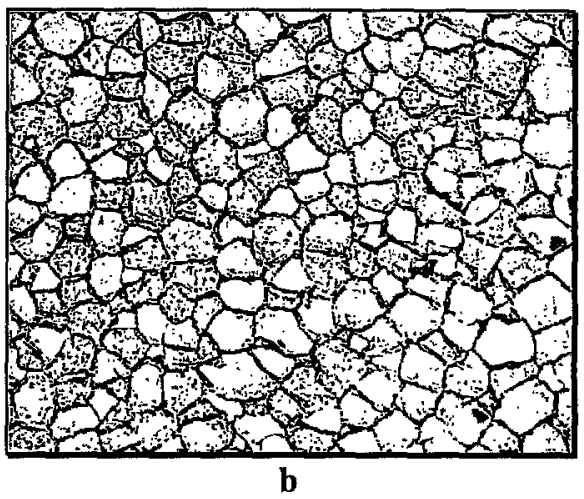

Fig. 3. Image from Fig. 1a binarized based on grey level histogram [5] (a) and with proposed technique (b).

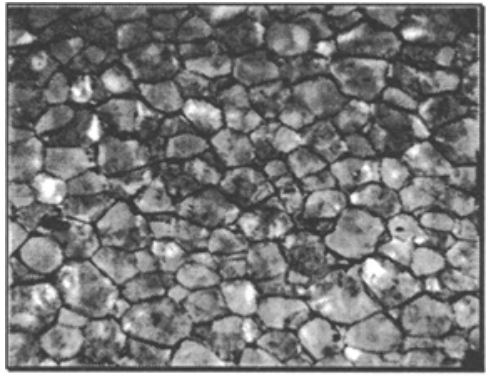

a

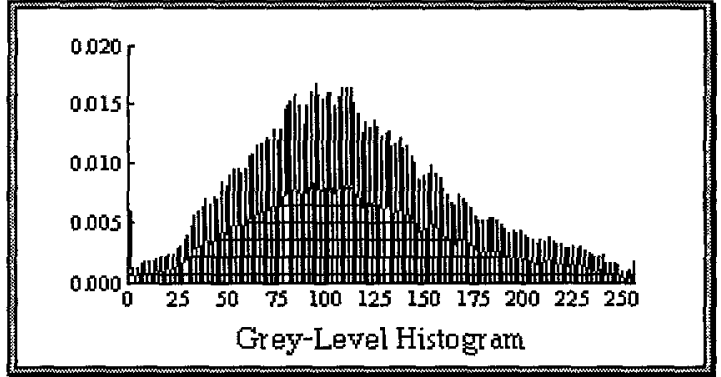

b

Fig. 4. Original image (a) and its grey level histogram (b).

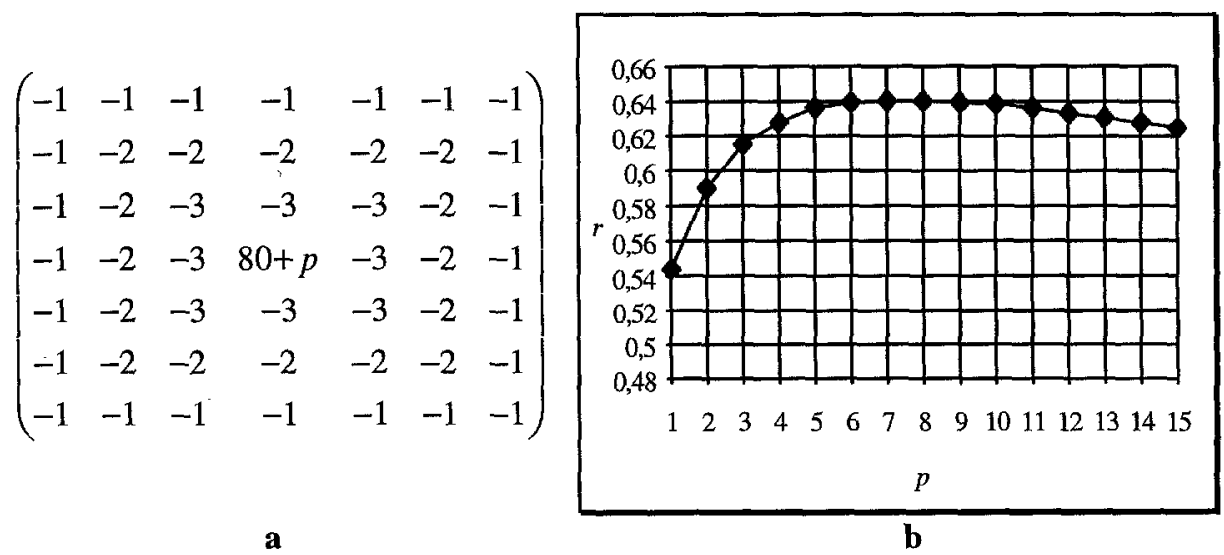

Fig. 5. Convolution kernel (a) and graph with the coefficient of correlation between original (Fig. 4a) and convoluted (with kernel from Fig. 5a for $p=1,2, \ldots, 15$ ) images vs. parameter $p$ (b). 

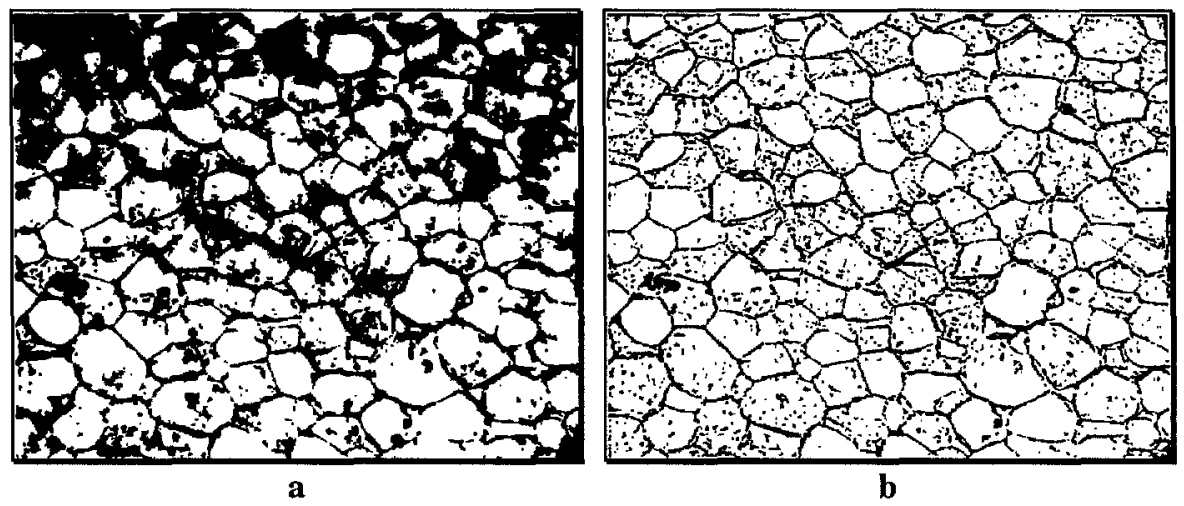

Fig. 6. Image from Fig. 4a binarized based on grey level histogram [5] (a) and with proposed technique (b).

\section{Conclusion}

A new binarization method based on discrete convolution filtering is developed. The algorithm is relatively simple to implement with low computational cost.

Examples of its application for binarization of images with inhomogeneous illumination are given. A comparison with histogram based threshold selection method is presented.

\section{References}

1. P.K. Sahoo, S. Soltani, A.K.C. Wong: A survey of thresholding techniques. Computer Vision, Graphics, and Image Processing 41, 233-260 (1988)

2. J.R. Parker: Gray level thresholding in badly illuminated images. IEEE Transactions on Pattern Analysis and Machine Intelligence 13, 813-819 (1991)

3. W. Niblack: An Introduction to Digital Image Processing. Englewood Cliffs, N.J.: Prentice-Hall International 1986

4. Anil K. Jain: Fundamentals of Digital Image Processing. London: Prentice-Hall International 1989

5. A.D. Brink: Gray-level thresholding of images using a correlation criterion. Pattern Recognition Letters 9, 335-341 (1989) 